journal club

\title{
Herz und Hirn profitieren von einer aggressiveren Blutdrucksenkung
}

Fragestellung: Ist eine aggressivere Blutdrucksenkung bei Patienten mit arterieller Hypertonie besser geeignet, kardiovaskuläre und renale Ereignisse zu reduzieren?

Hintergrund: Die arterielle Hypertonie ist ein wichtiger Risikofaktor für den Schlaganfall und kardiovaskuläre Erkrankungen. Die arterielle Hypertonie ist auch ein häufiger Grund für eine Niereninsuffizienz. Die bisherigen Leitlinien empfehlen systolische Blutdruckwerte unter 140/90 $\mathrm{mmHg}$ anzustreben und bei Patienten mit Diabetes mellitus unter 130/85 mmHg. Die 8. Joint National Commitee Guidelines empfehlen sogar bei über 60-Jährigen eine Grenze des Blutdrucks von 150/90 mmHg. Diese Empfehlungen sind aber keineswegs unumstritten.

Patienten und Methodik: Die Autoren führten eine systematische Literatursuche durch und identifizierten Studien mit denen eine aggressivere mit einer weniger aggressiven blutdrucksenkenden Therapie über einen Zeitraum von mindestens sechs Monaten verglichen wurden. Anschließend führten sie eine Metaanalyse durch: Endpunkte waren schwerwiegende kardiovaskuläre Ereignisse wie Herzinfarkt, Schlaganfall, Herzinsuffizienz und kardiovaskulärer Tod. Außerdem wurden die Gesamtsterblichkeit, die Entwicklung einer Niereninsuffizienz, schwerwiegende unerwünschte Arzneimittelwirkungen, Albuminurie und fortschreitende Retinopathie bei Patienten mit Diabetes mellitus ausgewertet.

Xie X, Atkins E, Lv J et al. Effects of intensive blood pressure lowering on cardiovas-cular and renal outcomes: updated systematic review and meta-analysis. Lancet 2016; 387: $435-43$
Ergebnisse: Die Autoren fanden 19 Studien mit insgesamt 44.989 Teilnehmern. Während der Studiendauer traten 2.496 schwerwiegende kardiovaskuläre Ereignisse auf,
1.762 Todesfälle und 514 Fälle eines Nierenversagens. Fast alle Studien waren offen. Die mittlere Beobachtungsdauer betrug 3,8 Jahre. Fünf Studien schlossen nur Patienten mit Diabetes mellitus ein. Sechs Studien umfassten Patienten mit chronischer Niereninsuffizienz. Zwei Studien rekrutierten Patienten mit Diabetes mellitus ohne arterielle Hypertonie.

Der mittlere Blutdruck in der weniger aggressiven Behandlungsgruppe lag im Mittel bei 140/81 mmHg, bei der aggressiv behandelten Gruppe bei 133/76 mmHg. Die relative Risikoreduktion zugunsten der aggressiveren blutdrucksenkenden Therapie betrug $14 \%$ für schwerwiegende kardiovaskuläre Ereignisse, $13 \%$ für Myokardinfarkte, $22 \%$ für Schlaganfälle, $10 \%$ für eine Albuminurie und 19\% für ein Fortschreiten einer vorbestehenden Retinopathie. Keine eindeutigen Auswirkungen der intensiven blutdrucksenkenden Therapie fand sich bei der Herzinsuffizienz mit einer relativen Risikoreduktion von $15 \%$, beim kardiovaskulären Tod mit $9 \%$, bei der Gesamtsterblichkeit von $9 \%$ und dem Nierenversagen mit $10 \%$. Die Analyse zeigte auch, dass es einen eindeutigen Nutzen einer blutdrucksenkenden Therapie gibt, wenn der systolische Blutdruck unter $140 \mathrm{mmHg}$ gesenkt wird. Der Nutzen der antihypertensiven Therapie war am größten bei Patienten mit vorbestehender vaskulärer Erkrankung, Nierenerkrankung oder Diabetes mellitus.

Schwerwiegende unerwünschte Arzneimittelwirkungen waren bei aggressiver und weniger aggressiver blutdrucksenkender Therapie nicht unterschiedlich. Lediglich eine klinisch wirksame arterielle Hypotonie fand sich beim aggressiven blutdrucksenkenden Regime häufiger.

Schlussfolgerungen: Eine aggressivere blutdrucksenkende Therapie senkt das Risiko von kardiovaskulären Ereignissen, Schlaganfall und vaskulärem Tod gegenüber einer weniger aggressiven Therapie.

\section{- Kommentar von Hans-Christoph Diener, Essen}

\section{Blutdrucksenkende Behandlung lohnt sich definitiv}

Diese große Metaanalyse zeigt sehr überzeugend, dass es sich definitiv lohnt, eine arterielle Hypertonie aggressiv zu behandeln. Soweit das von der Toleranz der Therapie bezüglich $\mathrm{Ne}-$ benwirkungen her vertretbar ist, gilt damit weiterhin das $\mathrm{Pa}$ radigma je niedriger umso besser. Das gilt vor allem für die Reduktion von Schlaganfällen und kardiovaskulären Ereignissen. Deutlich geringere Auswirkungen hat die aggressive Blutdrucksenkung auf die Entwicklung und Progression einer Herzinsuffizienz.

Bemerkenswert ist, dass die höchste Risikoreduktion für das Auftreten von Schlaganfällen erreicht wird. Eine interessante Beobachtung der Studie war, dass der Effekt einer besseren
Blutdrucksenkung über alle Patientengruppen hinweg zu beobachten war. Im klinischen Alltag in Deutschland besteht allerdings das Problem, dass bei vielen Patienten eine arterielle Hypertonie nicht diagnostiziert wird und selbst wenn sie bekannt ist, nicht ausreichend behandelt wird. Solange Patienten keine Beschwerden haben und kein vaskuläres Ereignis erlitten haben, ist die Einsicht in eine Notwendigkeit der Behandlung einer arteriellen Hypertonie bedauerlicherweise nicht sehr hoch.

Die Situation ist natürlich stark verändert, wenn Patienten bereits einen Endpunkt wie einen Myokardinfarkt oder einen Schlaganfall oder eine Hirnblutung erlitten haben. 